\title{
Eva Marinai
}

\section{Train de vie. II mirnggio proibito dell'Occidente nel teatro di Matei Vișniec}

\section{TRAIN DE VIE. THE FoRBIDDEN MIRAGE}

\section{OF THE West In MATEI VișNiec's Theatre}

Abstract: The subversive spirit of Matei Vișniec moves around the land of the forbidden and the censored. In Occidental Express (2009) the revolutionary idea takes the shape of a train of hope, heading to Paris, a heavenly mirage for migrants blinded by the lights on the horizon. Starting from the analysis of this sequence of tableaux vivants, this paper aims to reflect on the relationships that binds Utopia to postdramatic writing methods. Utopia is to be understood as a possibility for the intellectual to defeat the power relationships linking the consumerist West and the austerity of the communist regime, but also as a check suffered by the intellectual himself when he tries to overstep the laws of the system.

Keywords: Matei Vișniec; Postdramatic Theatre; Western Society; Revolution; Subversion; Contemporary Dramaturgy.

\section{Eva MARINAI}

Università di Pisa, Italia eva.marinai@unipi.it

DOI: $10.24193 /$ cechinox.2020.39.19
Tl teatro di Matei Vișniec, riconosciuto come modello di resistenza alla manipolazione ideologica, anticipa - come sempre accade alle arti - rivolgimenti politici e mutamenti culturali in una tensione continua e incessante tra realtà e utopia, ponendosi quale punto di osservazione privilegiato dell'accadere ${ }^{1}$. Un'osservazione critica che investe sia il suo paese natale, la Romania comunista soggetta al regime di Ceaușescu, sia le democrazie occidentali, essendo egli accolto come rifugiato politico in Francia nel 1987.

Intimidazioni, censure e pressioni non hanno intaccato lo spirito sovversivo di Vișniec, che d'altra parte non si è mai neppure lasciato toccare dalla nostalgia dell'esule né sedurre dalle lusinghe del liberismo occidentale.

La sua penna sottile, che non intinge mai nell'inchiostro puramente e ricercatamente letterario, abbozza scene plastiche e schegge di vita su cui si proietta lombra dell'autore, libero di vagabondare come un eroe tragico tra le tombe dei morti. Personaggi-funzione, i suoi, che vivono vite sospese, in attesa di qualcosa che non accadrà mai: il riferimento a Beckett è ormai 
scontato e più volte segnalato dagli studiosi che si sono avvicinati all'opera di Vișniec.

Peraltro, prima di Beckett e Ionesco ${ }^{2}$, il drammaturgo scopre attraverso Čechov l'interesse per una testualità teatrale polifonica e precorritrice del postmoderno (Guccini parla di «un'essenziale - e quasi cechoviana - levità di stile» ${ }^{3}$ ), tale da far supporre la presenza di una scrittura-collage che attinge a varie opere degli autori sopracitati (da Tre sorelle al Rinoceronte passando per Godot), numi tutelari per l'immaginazione del drammaturgo rumeno.

La creatività di Vișniec, ancorché imbevuta delle sollecitazioni absurdiste e surrealiste provenienti dall'alveo culturale francese (Camus in primis), tuttavia mai piegata a una logica nichilista, rispecchia la tensione verso una, seppur inattuabile, ricomposizione degli aspetti più contraddittori della società contemporanea. Mentre Camus acceca Meursault - e i suoi lettori - con i raggi abbaglianti del sole algerino e lo fa agire come in preda a un sonno della ragione, Vișniec sembra voler spalancare gli occhi dei suoi lettori/spettatori con il metodo usato nei confronti del protagonista di A Clockwork Orange di Burgess, affinché possano rendersi conto della violenza e degli orrori che albergano nella società in cui vivono.

Pur non essendo Vișniec un metteur en scène i suoi drammi contengono attributi e «aperture» assimilabili a esempi contemporanei di «scrittura scenica» ${ }^{4}$, presupposto intrinseco della nozione di postdrammatico individuato da Hans-Thies Lehmann ${ }^{5}$, sviluppando e oltrepassando il concetto di crisi del dramma moderno (Szondi). Ambigua e aperta è la nozione di scrittura scenica, che in senso lato chiama in causa l'autonomia dell'evento spettacolare in sé, non più considerato un semplice momento di illustrazione e verifica del testo drammatico.

È il lavoro «sul campo», che si tratti di libera invenzione o di rielaborazione/ riscrittura, a produrre la messinscena: massima è la centralità dell'attore, con la sua corporeità; ed estrema anche la variabilità dello spettacolo, che attraversa tante fasi evolutive quante sono le repliche. Matei Vișniec è dunque uno scrittore di teatro che conosce la materialità della scena e il corpo degli attori che incarnano le presenze e le voci che egli immagina; e che, in aggiunta, è riuscito ad affrancare la scrittura drammatica da quella dipendenza dalla letteratura che già il conterraneo Marin Sorescu denunciava provocatoriamente come uno dei mali necessari all'accettazione del genere in ambito culturale rumeno.

Peraltro, sempre Guccini nel suo saggio introduttivo a Drammi di resistenza culturale, mettendo in dialogo le riflessioni di Taviani, Meldolesi e Ferrone in merito, rispettivamente, alle potenzialità performative del testo teatrale, al ruolo del Dramaturg e alla differenza tra scrittura preventiva e consuntiva ${ }^{6}$, ha posto la questione del complesso rapporto tra processo compositivo, esito testuale e progetto spettacolare ${ }^{7}$. Una riflessione che investe il teatro tout court prendendo come spunto il caso Vișniec e che, oltrepassando l'ormai obsoleta rivendicazione dell'identità teatrale, restituisce onorabilità e autonomia alla drammaturgia d'autore, senza ingaggiare insensate e inutili battaglie, più ideologiche che concrete, con la drammaturgia d'attore. Concluso il Novecento, infatti, scrivere per il teatro dopo Beckett, come titola Lorenzo $\mathrm{Mango}^{8}$, significa anche affrontare le questioni relative alla posizione rivestita dalla scrittura letteraria laddove «affermare una netta dicotomia tra 
testualità letteraria e testualità scenica [ha] più un portato ideologico che reale»?

La forza di penetrazione dell'opera di Vișniec nel panorama internazionale, in particolare tra le giovani generazioni, è dovuta principalmente alle peculiarità della sua scrittura: una modalità ideativa e compositiva rivolta alle problematiche della contemporaneità veicolate attraverso un linguaggio aggiornato ai tempi, multiforme, impregnato di una miscela di corporeità e lirismo, epicità e drammaticità, evocazioni letterarie e materialità scenica, privo di psicologismi eppure in grado di delineare situazioni emotivamente coinvolgenti. La sensazione che si prova leggendo i drammi di Vișniec, ancor prima di vederli in scena, è quella di essere di fronte al mondo che ci circonda, che si conosce bene, ma visto attraverso una lente d'ingrandimento che isola i dettagli deformando l'insieme e costringendo il lettore/ regista/spettatore a un lavoro di decodifica e ricomposizione, come accade in una ripresa in cui prevalgano primi piani e particolari. Lo strumento di precisione con cui l'autore riesce a sezionare chirurgicamente il reale è la sua «scrittura acuminata ${ }^{10}$, in grado di «superare le barriere linguistiche»; e ancora, una scrittura come «scontro frontale. Come in un incidente... si viene investiti e non si può far finta di niente» ${ }^{11}$.

Come registra Emilia David, tale «poetica modulare» suggerisce esplicite analogie con la pittura cubista e con il montaggio scenico o cinematografico, che permettono «un modello non gerarchico dei segni teatrali» e «una percezione sinestetica di ogni particolare visivo» ${ }^{12}$. Già Georges Banu, introducendo l'edizione francese del Teatro decomposto o l'uomo pattumiera $^{13}$, aveva evidenziato un'analogia tra la «modularità» di Vișniec e i tableaux di un'esposizione d'arte che lasciano libertà al visitatore/lettore/spettatore di ricostruire in autonomia la propria «logica visiva»:

L'auteur livre des matériaux en invitant le metteur en scène à construire lui-même son organisation, à les enchaîner et alterner selon une logique à inventer chaque fois. Cela n'est pas sans rappeler l'état de cette œuvre de la modernité Woyzeck de Büchner dont les fragments peuvent être distribués selon le scénario élaboré par le metteur en scène qui s'engage à les montrer ${ }^{14}$.

L'insieme di questi aspetti, congiuntamente alle potenzialità espressive di pièce che, salvo rare eccezioni, coinvolgono un numero piuttosto elevato di attori, rende il teatro di Vișniec adatto a ensemble e grandi compagnie, molto rare nel panorama professionistico (soprattutto italiano), perlopiù formato da solisti e micro-formazioni, spesso con genesi autodidatta e refrattaria al testo tradizionale, oppure orientato alla messa in scena di drammi canonizzati. Perciò, per quanto concerne in particolare il nostro Paese (ma anche in Francia), milieu teatrale privilegiato dellopera di Vișniec è quello sperimentale, laboratoriale e delle scuole di teatro, solitamente assetato di buona drammaturgia contemporanea, aperto alle sollecitazioni et(n) iche provenienti dai Sud-Est del mondo e nondimeno capace di mettere alla prova le competenze registiche e attoriche degli allievi. Non è un caso che Giorgio Strehler sia stato il primo, in Italia, a guardare ai testi di Vişniec per il suo Teatro Studio nei primi anni Novanta. 
Nel 1994 il giovane regista rumeno Ștefan Iordănescu, già direttore del teatro di Timișoara e con alle spalle maestri come Bergman, Brook e lo stesso Strehler, si fa conoscere al Festival dei Teatri d'Europa durante un workshop con alcuni allievi attori del Piccolo Teatro di Milano svolto sul testo del coetaneo Matei Vișniec, Vecchio clown cercasi. E in quelloccasione che Strehler propone a Iordănescu di trasformare quel primo esercizio in un vero allestimento da portare all'attenzione del pubblico italiano.

Quattro anni dopo lo spettacolo debutta al Teatro Studio di via Rivoli, all'intero del progetto strehleriano "Giovani Registi Europei», vetrina per talenti emergenti e per attori formati alla pionieristica Scuola del Piccolo, alla quale il regista milanese proprio in quegli anni cercava di dare un'ampia visibilità e una precisa identità politica interculturale ${ }^{15}$.

Il mondo «in maschera» di Vecchio clown cercasi, che per certi aspetti sembra arieggiare il cinema di Fellini, la pittura di Francis Bacon e naturalmente il miraggio, l'illusione e l'eterna attesa beckettiana, ha segnato il primo contatto dell'opera drammaturgica di Vișniec con il mondo teatrale italiano $^{16}$.

A questa altezza cronologica il drammaturgo di Rădăuți aveva già conosciuto un primo successo di pubblico e critica con Cavalli alla finestra per la regia di Pascal Papini, in occasione delle «Journées des Auteurs» organizzate dal Théâtre les Célestins di Lione (1992); i suoi testi avevano incominciato a transitare dalle maggiori piazze francesi (Parigi, Marsiglia, Tolosa, Nizza) approdando nella sezione Off del Festival d'Avignone, che oggi annovera nel complesso più di quaranta allestimenti delle sue opere ${ }^{17}$.
In generale, lopera di Vișniec suscita le più diverse attenzioni da parte del panorama teatrale europeo, ma si può riscontrare un interesse specifico in quei territori di confine e in quei contesti particolarmente sensibili alle problematiche sociali, al fenomeno dei flussi migratori e dell'interazione tra culture differenti ${ }^{18}$.

È così anche nel caso di Occident $[a l]$ Express, il cui debutto, in Italia come in Francia, avviene nell'ambito di progetti di cooperazione internazionale. È finanziata dal Fondo Sociale Europeo e ha parte della troupe proveniente dalla città rumena di Arad la versione di Gianpiero Borgia data in prima nazionale il 23 ottobre 2011 al Teatro Curci di Barletta.

Mentre il regista Cyril Ripoll, con i giovani attori della compagnia Les Anciens de Barbusse, presenta la medesima pièce nel corso dell'edizione 2014 del «Festival des écritures» di Alfortville, popolosa città a sud di Parigi con una ormai radicata presenza di immigrati armeni. Barletta e Alfortville: terre di sbarco, di attraversamenti e nuove ripartenze. Questo aspetto è particolarmente significativo perché mette in relazione la biografia personale dell'esule Vișniec, la sua poetica e il contesto ricettivo della sua opera. Nondimeno, attribuire ai suoi lavori la sola etichetta di «teatro di interazione sociale» potrebbe apparire una diminutio. È necessario invece considerare tale modalità di penetrazione nel tessuto teatrale internazionale come una forma di sdoganamento, uno dei possibili lasciapassare per l'accreditamento di autori e opere che altrimenti faticherebbero a raggiungere i canali della produzione e distribuzione spettacolare.

Con Occidental Express $(2009)^{19} \mathrm{gli}$ avvertimenti politici di Vișniec assumono sostanza drammaturgica nella forma di un 
treno della speranza diretto a Parigi, miraggio paradisiaco per migranti accecati dalle luci all'orizzonte: un avvicendamento di 13 scene, autosufficienti ma agganciate a uno stesso binario (per insistere sulla metafora dei vagoni di un convoglio), che si snodano senza una precisa successione cronologica e dove l'ironia costituisce l'elemento retorico dominante.

La continuità è rappresentata da vari elementi di carattere semantico e strutturale, tra cui il più evidente è forse costituito dalla coincidenza tra la scena di chiusura e la scena di apertura. L'anziano padre cieco sulla sedia a rotelle (già paragonato a Edipo da Gerardo Guccini ${ }^{20}$ ), il quale, in una stazione polverosa, vuole toccare, come si tocca un oggetto del desiderio tanto appetibile quanto più inarrivabile, lo storico Orient Express di ritorno da Bucarest verso Parigi. Il treno - che non passa e non passerà mai da quella stazioncina rumena - incarna, nell'immaginario collettivo evocato da Vișniec, l'utopia del crollo dei muri, dei regimi totalitari e della cortina comunista, ma anche, volendo leggere tra le righe, la critica alla degenerazione del sistema sociale e dunque alle insidie nascoste dietro la democrazia (dove «l'identificazione del male è più difficile» ${ }^{21}$ ).

Scrivendo che:

la follia o la minaccia nazionalista non sono soltanto appannaggio dei paesi poveri, sotto-sviluppati, recentemente usciti da una lunga dittatura. Questa follia può colpire e scavare dei fossati psicologici, etnici, sociali anche nei paesi ricchi che hanno una lunga tradizione democratica ${ }^{22}$,

l'autore rende biunivoca la direzione degli sguardi: i sentimenti di speranza, rivendicazione, finanche invidia per un benessere non condiviso, che muovono un individuo e una comunità intera verso un territorio diverso da quello natio, hanno sempre un corrispettivo, spesso conflittuale, nei sentimenti di tenace resistenza, avidità, indisponibilità provati da un'altra comunità; proprio come un doppio binario che consente l'andata e il ritorno di un convoglio.

E utile, a questo punto, riflettere sul sistema di relazioni che lega l'Utopia alle modalità di scrittura postdrammatica. Utopia intesa, nel caso specifico, come vittoria dell'intellettuale sui rapporti di potere tra il miraggio dell'Occidente consumistico e l'austerità del regime comunista; ma anche come scacco subito dallo stesso intellettuale nel momento in cui cerca di sconfinare dalle leggi del sistema di appartenenza.

L'elemento utopico - e parimenti postdrammatico - del teatro di Vișniec può essere letto con una prospettiva duplice. Da un lato, quello più specificamente formale, l'utilizzo di un linguaggio franto, di matrice cinematografica - nella misura in cui fa ampio uso della tecnica del montaggio - con un intreccio dialettico tra ipotesti e ipertesti (pastiche), uso della paratassi e della plurivocalità, anonimato dei personaggi, stratificazione e frammentazione dei contenuti, fusione dei registri, intertestualità.

Dall'altro, la volontà di veicolare - pur evitando intenti moraleggianti - contenuti dalla forte matrice politica attraverso l'allusione e la metafora, strumenti elettivi per chi voglia eludere i meccanismi della censura. Si tratta di un modus operandi inevitabilmente legato alla biografia e al percorso artistico ed esistenziale dell'autore, che ha attraversato il regime di Ceaușescu, il crollo del comunismo nel paese natale e 
il conseguente approdo nella Francia anni Ottanta.

E proprio il movimento intellettuale della Generazione Ottanta, di cui l'autore fa parte e che ha contribuito a fondare, che traghetterà la cultura rumena verso il postmodernismo, praticando una nuova forma di scrittura basata sul rifiuto del realismo socialista e delle estetiche ermetiche e formaliste dell'avanguardia, annunciando invece una vocazione alla poetica del quotidiano, della presa diretta della realtà, tesa alla demistificazione del sistema di propaganda, attraverso l'uso di una retorica tragicomica e allegorica per sfuggire alla censura:

Piuttosto che accettare un compromesso con il partito, la Generazione Ottanta preferisce vagabondare tra le strade della miseria armandosi di telecamera e registratore, incontrando povertà, carestie e oppressione, per poter trarre da fonti attendibili i canovacci dei propri racconti. Le loro armi per eludere la censura e demistificare il sistema sono le metafore, le allusioni, l'ironia ${ }^{23}$.

La censura è ingannata anche attraverso una sostituzione della parola con il gesto: un gesto scenico, che può addirittura ribaltare la prospettiva testuale intridendo di ironia espressioni apparentemente prive di potere sovversivo. Si tratta di un meccanismo retorico usato frequentemente dagli autori di teatro politico-satirico ad ogni latitudine e altezza cronologica, da Aristofane in poi. Una partitura gestuale, quella delineata dal teatro di Vișniec, che, come tale, era difficilmente rintracciabile dal controllo preventivo sui testi (come afferma egli stesso: «Non erano le parole a contare, quelle erano accettate perché stavano nei libri, erano 'classiche'. I gesti invece erano pericolosi ${ }^{24}$ ).

L'autore racconta i motivi per cui la censura attaccò ferocemente il teatro più di ogni altra pratica letteraria o artistica:

Molto spesso mi sono chiesto perché in Romania il teatro fosse più censurato dei romanzi e della poesia. Il fatto è che il teatro faceva più paura di un romanzo di denuncia. Per il potere totalitario, un libro non era pericoloso quanto uno spettacolo perché il libro è letto in solitudine. $\mathrm{E}$ anche se il libro spinge alla rivolta, non puoi uscire da solo in strada a manifestare. Invece uno spettacolo che provochi un'emozione collettiva in quattrocento spettatori e trenta attori può creare una rivolta subito! Questa è la ragione per la quale il teatro era più censurato rispetto agli altri generi. [...].

C'era una grande complicità tra il pubblico e gli artisti. Il regista lavorava con immagini allusive e il pubblico sapeva come decodificare i suoi messaggi nascosti ${ }^{25}$.

In effetti, a ben pensarci, le due prospettive sopra delineate si rassomigliano fino a potersi dire congruenti, soprattutto se ci poniamo dal punto di osservazione dell'bomo faber, dell'artista creatore di mondi.

Il teatro, producendo nell'atto presente, cioè non in forma puramente teorica, astratta o grafica, un legame credibile tra elementi eterogenei è di per sé utopico. Peculiare nel caso di Vișniec è - e in ciò si riassume la doppia prospettiva di cui sopra - il tentativo, a un tempo etico ed estetico, di disegnare una realtà meno volgare di 
quella quotidiana. Ciò avviene, come si è detto, sia sul piano stilistico, con i presìdi creativi della trasformazione, della decomposizione e della condensazione delle strutture linguistiche, sia sul piano della situazione drammatica.

Qui scendiamo nel particolare sviluppo delle sequenze sceniche di Occidental/Occident Express che, sebbene non siano traverse di una stessa strada, portano comunque questa connotazione semantica: ciò che non vediamo è migliore di ciò che vediamo, proprio perché non lo vediamo. E la frontiera, limen tra ciò che è di fronte ai nostri occhi e ciò che ci è precluso, è un confine su cui urinare (come fa il cieco nella scena quinta) non solo e non tanto per marcare il territorio, ma per appropriarsi, volgarizzandola, dell'idea astratta che il mondo oltre la siepe evoca. Con un'interpretazione «etologica», non è il gesto dell'animale che vuole circondare il suo ambiente con il suo stesso odore, per il bisogno di sentirsi più al sicuro, ma indica semmai il disdegno per una convenzione tanto illogica quanto cogente.

Il personaggio del cieco diviene quindi paradigma dell'utopia contemporanea, diretta conseguenza della società del consumo, proprio perché le immagini mentali che egli descrive (le carrozze di lusso, il vagone ristorante, il profumo) divengono più realistiche del reale. Si tratta dello stesso meccanismo che l'autore adotta nelle opere teatrali che precedono Occidental Express, da Teatro decomposto - L'uomo pattumiera a La storia del comunismo raccontata ai malati di mente, fino a La parola 'progresso' sulla bocca di mia madre suonava terribilmente fals ${ }^{26}$. Anche qui la «resistenza culturale» dell'autore si manifesta attraverso una lotta contro l'alienazione dettata da una convulsa ed estrema razionalizzazione (il formalismo come arma di distruzione di massa), mentre la follia ne costituisce l'unica via di fuga.

In La storia del comunismo raccontata ai malati di mente, lo scrittore, compagno stalinista, Yuri Petrowski prova così il suo discorso ai ricoverati nell'Ospedale psichiatrico di Mosca:

Aprite bene la bocca. Dite 'u'. Respirate. Riempite d'aria i polmoni. Più forte. Ancora. Riempite bene i polmoni d'aria. Ancora. Dite 'utopia'. Ancora una volta. 'Utopia'. Concentratevi bene, è una parola che fa una curva ascendente. È come un cavallo che si impenna. 'Utopia'. Sentite come salgono i suoni? Salgono e si dissolvono nell'aria. Tutto nasce nella vostra bocca e non finisce da nessuna parte. 'Utopiiiia'. Benissimo ${ }^{27}$.

La risposta dei malati sarà perlopiù volta a destabilizzare con azioni concrete e infantilmente sovversive la teoresi governativa: «Voglio pisciare in piedi!», dirà Ivan, uno dei ricoverati.

Peraltro, i personaggi monologanti e monomaniaci scaturiti dall'inventio dell'autore traspongono in termini visionari e talvolta smarriti questioni del presente, ma che finiscono poi per aprire uno squarcio sul rapporto deviato che l'essere umano intrattiene con le grandi questioni legate al senso del proprio esistere: l'amore, la morte, la paura, l'isolamento (esistenziale e geografico). L'Occidente lontano, «incorporeo, mitico, mentale ${ }^{28}$, emerge in quasi tutte le scene che compongono l'opera e s'incarna ora nel treno della speranza diretto a Parigi, ora nel miraggio 
dei soldi facili, come quelli che le ragazze madri alla frontiera cercano di estorcere ai soldati americani prostituendosi.

Nessun intento moraleggiante, a conferma del carattere postmoderno e postdrammatico del teatro di Vișniec, bensì una descrizione nitida e affilata delle contraddizioni insite nellesistente: come nella scena quarta aggiunta alla riscrittura di Occident Express, dove immigrati rumeni lavorano a Torino per costruirsi in Romania case che non abiteranno mai e che resteranno vuote, occupate dai cani e dalla loro urina (perfino gli attici!), e destinate soltanto a essere mostrate sugli schermi dei cellulari. Una pièce, questa, emblematica del miraggio economico dell'Occidente, che non riempie alcun vuoto, anzi ne produce altri. Come Vișniec fa dire polemicamente al personaggio 5 in questa scena: «il capitalismo $[\ldots]$ è una scienza esatta, l'equilibrio tra il troppo pieno e il vuoto». Sovrabbondanza ed eccesso da un lato, penuria e bisogno dall'altro: questo è l'Assurdo.

D'altra parte, la scena-refrain che vede un candidato discutere la propria originale tesi di dottorato sulla Romania iper-colonizzata di fronte a una commissione di sconcertati e ostili accademici diviene rappresentativa e rivelativa per lo stile con cui rimodula l'uso del nonsense (come scrive Emilia David: «lesperienza quotidiana dell'assurdo che ha pervaso nel modo più concreto l'esistenza del popolo rumeno $»^{29}$ ), costruendo una scrittura scenica intrisa di amara ironia.

Si potrebbe dunque concludere che il teatro di Vișniec adotti uno sguardo anamorfico, laddove nellopera sono nascosti significati alternativi. E indubbio, infatti, che la parte di mondo che egli intende rappresentare, nella sua apparente linearità, dischiude un orizzonte ambiguo e sfaccettato, che è possibile decifrare solo allontanando il punto di vista e allargando il cono prospettico. In questo modo si comprende come il rapporto tra l'autore e il potere, che è anche e soprattutto potere di distorcere la realtà, sia un gioco di forza: se chi comanda deforma la realtà, l'artista ha il potere (e il dovere) di ricrearla attraverso un'allucinazione, una "percezione senza oggetto». Il potere metamorfico della creazione teatrale è più forte della falsificazione della Storia. E Matei Vișniec è capace di fare questo, di dar vita a immagini e creature interiori attraverso la modalità peculiare degli uomini di teatro, ossia facendo dialogare più mezzi e contenuti espressivi entro un medesimo ambito culturale, che Emilia David ha definito perfettamente come «potenziale espressivo polivalente» ${ }^{30}$ : 1'adozione di un logos altro (in questo caso il francese), percepito beckettianamente come territorio vergine, la visualità (anche grafica e bozzettistica), il suono della parola, il corpo del testo, il gesto performativo, la forza delle idee, e infine la libertà di pensiero.

\section{Bibliografia}

Bartolucci, Giuseppe, La scrittura scenica, Roma, Lerici, 1968.

Bartolucci, Giuseppe, Teatro-corpo, teatro-immagine (per una «materialità della scrittura scenica»), Padova, Marsilio, 1970.

David, Emilia, «L'estetica del nuovo linguaggio drammaturgico nella poetica del 'teatro decomposto' di Matei Vișniec», Introduzione a Matei Vișniec, in 'La storia del comunismo raccontata ai malati di mente' $e$ altri testi teatrali, a cura di Emilia David, Spoleto (PG), Editoria\&Spettacolo, 2012, p. 5-30. 
David, Emilia, «L'assurdo della dittatura riletto da Matei Vișniec nel 'teatro dell'assurdo' di Eugène Ionesco», in Biblioteca Teatrale, n. 127-128, luglio-dicembre 2018, numero monografico dedicato al tema Generazioni a confronto. Eredità persistenze, tradizioni e tradimenti sulla scena moderna e contemporanea, a cura di Anna Barsotti, Erica Magris ed Eva Marinai, Roma, Bulzoni, 2018, p. 191-208.

Ferrone, Siro, «Drammaturgia e ruoli teatrali», in Il castello di Elsinore, 1988, n. 3, p. 37-44.

Guccini, Gerardo, «Pensare i corpi. I teatri di Vișniec», saggio introduttivo a Matei Vişniec, Drammi di resistenza culturale. I Cavalli alla finestra, La Donna come campo di battaglia, trad. it. di Pascale Aiguier, Davide Piludu e Giuseppa Salidu, Corazzano (PI), Titivillus, 2009, p. 5-65.

Guccini, Gerardo e Claudio Meldolesi (a cura di), Prove di Drammaturgia, Anno XV, n. 1, 2009, numero monografico dedicato a Matei Vișniec.

Guccini, Gerardo, «Limprendibile treno della storia», in Matei Vișniec, Occidental Express, trad. it. di Gianpiero Borgia, Corazzano (PI), Titivillus, 2012.

Lehmann, Hans-Thies, Il teatro postdrammatico, trad. it. di Sonia Antinori, Imola (BO), Cue Press, 2017. Mango, Lorenzo, La scrittura scenica. Un codice e le sue pratiche nel Novecento, Roma, Bulzoni, 2001.

Mango Lorenzo, Il Novecento del teatro. Una storia, Roma, Carocci, 2019.

Meldolesi, Claudio e Renata Molinari, Il lavoro del dramaturg. Nel teatro dei testi con le ruote, Milano, Ubulibri, 2007.

Pavis, Patrice, Problème de sémiologie théâtrale, Les Presses de l'Université du Québec, 1976.

Taviani, Ferdinando, Uomini di scena, uomini di libro. Introduzione alla letteratura teatrale italiana del Novecento, Bologna, il Mulino, 1995.

Vișniec, Matei, Drammi di resistenza culturale. I Cavalli alla finestra, La Donna come campo di battaglia, trad. it. di Pascale Aiguier, Davide Piludu e Giuseppa Salidu, Corazzano (PI), Titivillus, 2009.

Vișniec, Matei, 'La storia del comunismo raccontata ai malati di mente' e altri testi teatrali, a cura di Emilia David, Spoleto (PG), Editoria\&Spettacolo, 2012.

Vișniec, Matei, Occidental Express, trad. it. di Gianpiero Borgia, con un saggio introduttivo di Gerardo Guccini, Corazzano (PI), Titivillus, 2012.

Vișniec, Matei, Sul lavaggio del cervello a Est e a Ovest, lettura pubblica di Thomas Otto Zinzi, VI edizione del Seminario culturale TransEuropaExpress, «Storie e identità. L'Europa degli intellettuali», Auditorium dell'Ara Pacis, Roma, 3-4 marzo 2011.

\section{Note}

1. Anche se pochi in Romania avrebbero scommesso sulla caduta del regime comunista prima della condanna di Ceaușescu nel 1989, Vișniec fa notare che nel Teatro Nazionale di Bucarest già da dieci anni si rappresentava il Caligola di Albert Camus con ampia e consapevole partecipazione di pubblico. Cfr. Matei Vișniec, «Il lavoro 'orizzontale’ dell'autore», in Prove di Drammaturgia, Anno XV, n. 1, 2009, p. 4.

2. Gerardo Guccini ci rammenta come l'autore rumeno, «debitore delle drammaturgie di Beckett e Ionesco, $[\ldots]$ colloc[hi] i personaggi in situazioni aperte: e cioè non predeterminate da plot o schemi d'intreccio»: Gerardo Guccini, «Pensare i corpi. I teatri di Vișniec», saggio introduttivo al volume Matei Vișniec, Drammi di resistenza culturale. I Cavalli alla finestra, La Donna come campo di battaglia, trad. it. di Pascale Aiguier, Davide Piludu e Giuseppa Salidu, Corazzano (PI), Titivillus, p. 16. La prima volta che una pièce di Vișniec viene rappresentata in Italia (Vecchio clown cercasi, per la regia di Ștefan Iordănescu, Teatro Studio di Milano, stagione 1997-98), la rassegna stampa cita Beckett: cfr. ad es. Antonio Calibi, «La vita è cosa da pagliacci. Il testo del rumeno Vișniec da stasera al teatro studio», in La Repubblica, 19-02-1998: «[...] le sue atmosfere grottesche e lunari rimandano a [...] Beckett soprattutto per la dilatazione temporale in un'attesa senza traguardi». Al debito di Matei Vișniec nei confronti del teatro di Ionesco Emilia David dedica un intero saggio: Emilia David, «L'assurdo della dittatura riletto da Matei Vişniec nel 'teatro dell'assurdo' di Eugène Ionesco», in Biblioteca Teatrale, n. 127-128, luglio-dicembre 
2018, numero monografico dedicato a Generazioni a confronto. Eredità persistenze, tradizioni e tradimenti sulla scena moderna e contemporanea, a cura di Anna Barsotti, Erica Magris ed Eva Marinai, Roma, Bulzoni, 2018, p. 191-208.

3. Gerardo Guccini, «Pensare i corpi. I teatri di Vișniec», p. 10.

4. Secondo i criteri delineati da Patrice Pavis (Problème de sémiologie théâtrale, Les Presses de l'Université du Québec, 1976), rielaborati in tempi più recenti da Lorenzo Mango (La scrittura scenica. Un codice ele sue pratiche nel Novecento, Roma, Bulzoni, 2001). La scrittura scenica, Roma, Lerici, 1968, e Teatro-corpo, teatro-immagine (per una «materialità della scrittura scenica»), Padova, Marsilio, 1970 sono due saggi fondamentali con i quali Giuseppe Bartolucci trasporta in Italia una nozione la cui paternità è da attribuire a Roger Planchon, quella di scrittura scenica, riconcettualizzata come condizione materiale che implica tutti i codici del linguaggio spettacolare.

5. Hans-Thies Lehmann, Il teatro postdrammatico, trad. it. di Sonia Antinori, Imola (BO), Cue Press, 2017.

6. Si fa qui riferimento ai seguenti testi: Ferdinando Taviani, Uomini di scena, uomini di libro. Introduzione alla letteratura teatrale italiana del Novecento, Bologna, il Mulino, 1995; Claudio Meldolesi e Renata Molinari, Il lavoro del dramaturg. Nel teatro dei testi con le ruote, Milano, Ubulibri, 2007; Siro Ferrone, «Drammaturgia e ruoli teatrali», in Il castello di Elsinore, 1988, n. 3, p. 37-44.

7. Gerardo Guccini, «Pensare i corpi. I teatri di Vișniec», p. 5-65, in particolare alle p. 5-10.

8. Lorenzo Mango, Il Novecento del teatro. Una storia, Roma, Carocci, 2019, p. 335.

9. Ibid.

10. Nicola Bonazzi, «Al lavoro su Del sesso della donna come campo di battaglia nella guerra in Bosnia», in Prove di Drammaturgia, n. 1, 2009, p. 31.

11. Pascale Aiguier in Pascale Aiguier e Davide Piludu, «Note di regia a Voci nel buio», in ibid., p. 30.

12. Emilia David, «L'estetica del nuovo linguaggio drammaturgico nella poetica del 'teatro decomposto' di Matei Vișniec», Introduzione a Matei Vișniec, in 'La storia del comunismo raccontata ai malati di mente' e altri testi teatrali, Spoleto (PG), Editoria\&Spettacolo, 2012, p. 10.

13. Georges Banu, «Matei Vișniec ou de la Décomposition», in Matei Vișniec, Théâtre décomposé ou l'homme-poubelle, Parigi, Institut Français de Bucarest-Éditions L'Harmattan, 1996, p. 7-9.

14. Ibid., p. 7.

15. Cfr. Archivio del Piccolo Teatro di Milano, che alla voce «Vecchio clown cercasi - 1997-98» include una scheda dello spettacolo (autore: Matei Vișniec; traduzione: Sabrina Faller; regia: Ștefan Iordănescu; con: Roberto Zibetti, Giorgio Bongiovanni, Luca Criscuoli; scene: Velica Panduru; costumi: Velica Panduru; musiche: Ildikó Iordănescu Fogarasi), la locandina, 23 immagini e una rassegna stampa di 19 articoli.

16. «L'atmosfera è lunare - continua il regista - perché la luce notturna dà un senso cosmico. I tre personaggi non sono solo clown di circo, sono Pierrot lunaire, astrazioni della Commedia dell'Arte, figure dalle doppie facce. Perché anche la morte ha una faccia meravigliosa», Antonio Calbi, «La vita è cosa da pagliacci. Il testo del rumeno Vișniec da stasera al teatro studio», citato in precendenza.

17. Cfr. a titolo esemplificativo, le sei messinscene che gli sono state dedicate nel 2018: https://www. courrierdesbalkans.fr/Festival-d-Avignon-6-pieces-de-Matei-Vișniec-dans-le-OFF (consultato il 25 luglio 2019).

18. Cfr. Gerardo Guccini, «Pensare i corpi. I teatri di Vișniec», p. 6, n. 1: «[...] alle rappresentazioni effettuate da giovani formazioni (il Teatro Laboratorio Alkestis di Cagliari e Mali Weil di Milano) si affiancano [...] le produzioni del Teatro Stabile di Catania (Come spiegare la storia del comunismo ai malati di mente con la regìa di Gianpiero Borgia) e della Compagnia Teatro dell'Argine, realtà di punta nell'elaborazione di linguaggi teatralmente fondati e aperti alle problematiche del sociale (Del sesso della donna come campo di battaglia nella guerra in Bosnia con la regìa di Nicola Bonazzi). Va segnalato, quale indizio della fortuna di questo autore nei Paesi dell'Europa orientale, che Irina Pușcaș e Mihail Lebeclenco, due attori/migranti di origine moldava che ora collaborano con il Teatro dell'Argine, si sono formati presso l'Accademia di Teatro, Musica e Arti Plastiche di Chișinău, recitando testi di Vișniec». 
19. Il dramma esce in Romania per i tipi dell'editore Paralela 45. Viene poi riscritto in francese con l'aggiunta di due episodi (uno sul lavoro degli immigrati rumeni in Italia e uno centrato sulla visita di un gruppo di turisti in Transilvania). Quest'ultima versione è apparsa nel 2020 per le edizioni Non lieu di Parigi, quando il presente saggio era in bozza: ringrazio l'autore ed Emilia David per avermi fornito la copia dattiloscritta ancora inedita del testo. La prima edizione italiana si avvale della traduzione del regista Gianpiero Borgia (funzionale alla messa in scena del 23 ottobre 2011, Teatro Curci di Barletta): Matei Vișniec, Occidental Express, trad. it. di Gianpiero Borgia, con un saggio introduttivo di Gerardo Guccini, Corazzano (PI), Titivillus, 2012.

20. Cfr. Gerardo Guccini, «L'imprendibile treno della storia», in Matei Vișniec, Occidental Express, trad. it. di G. Borgia, p. 8.

21. Cfr. Matei Vișniec, Sul lavaggio del cervello a Est e a Ovest, lettura pubblica di Thomas Otto Zinzi, VI edizione del Seminario culturale TransEuropaExpress, «Storie e identità. L'Europa degli intellettuali», Auditorium dell'Ara Pacis, Roma, 3-4 marzo 2011.

22. Cit. in Giuseppa Salidu (a cura di), «La Romania mi ha dato le radici, la Francia le ali», in Prove di Drammaturgia, n. 1,2009, p. 10.

23. Ibid., p. 6.

24. Matei Vișniec, «Il lavoro 'orizzontale' dell'autore», p. 2.

25. Ibid., p. 4.

26. Cfr. Matei Vișniec, 'La storia del comunismo raccontata ai malati di mente' e altri testi teatrali.

27. Ibid., p. 107.

28. Gerardo Guccini, «Limprendibile treno della storia», p. 9.

29. Emilia David, «L'estetica del nuovo linguaggio drammaturgico nella poetica del 'teatro decomposto' di Matei Vișniec», p. 7.

30. Ibid., p. 5. 